ORIGINAL ARTICLE

\title{
Pilot study of a paediatric emergency department oral rehydration protocol
}

\author{
R Boyd, M Busuttil, P Stuart
}

Emerg Med J 2005;22:116-117. doi: 10.1136/emj.2003.010801

See end of article for authors' affiliations

.....................

Correspondence to: Dr R Boyd, Department of Emergency Medicine, Lyell McEwin Health Services, Haydown Road, Elizabeth Vale SA 5112, South Australia; russellboyd@ nwahs.sa.gov.au

Accepted for publication 15 March 2004

\begin{abstract}
Objective: To ascertain if the use of a paediatric oral rehydration protocol in the emergency department changed rates of admission, total time spent in hospital, total time spent in the emergency department, or number of unscheduled returns to the emergency department.

Methods: A two month time period was analysed before and then after the adoption of an oral rehydration protocol for children presenting to the emergency department with symptoms suggestive of gastroenteritis. The rates of admission, total time spent in hospital, total time spent in the emergency department, and the rate of unscheduled returns were analysed using Fisher's exact and Wilcoxon testing for non-parametric data.

Results: The adoption of a paediatric rehydration protocol was associated with a significant reduction in admission rates (before $=22.5 \%$, after $5.1 \% ; p=0.048$ ) and mean total hospital time (before $=7$ hours 54 minutes, after $=2$ hours 17 minutes; $p=0.017$ ). There was no significant difference in time spent in the emergency department (before $=1$ hour 25 minutes, after 1 hour 35 minutes, $p=0.3$ ). The number of unscheduled returns did not change significantly $(p=0.3)$.

Conclusion: Adoption of a paediatric rehydration protocol significantly reduces admission rates and total time spent in hospital for children presenting to the emergency department with symptoms of gastroenteritis. The mean time spent in the actual emergency department does not significantly increase. The rate of unscheduled returns does not change.
\end{abstract}

$\mathrm{P}$ resentations to the paediatric emergency department with the symptoms of gastroenteritis, namely vomiting and diarrhoea are common in all healthcare systems. The typical response is that of assessment for dehydration or even circulatory collapse and some method of fluid replacement therapy. Increasingly, evidence suggests that for cases of mild to moderate dehydration the use of proscriptive oral rehydration strategies are effective. The current World Health Organisation guidelines suggest oral rehydration therapy as initial treatment for mild and moderate dehydration. ${ }^{2}$ In addition, when compared with intravenous fluid therapy, oral therapy has been shown to actually reduce morbidity ${ }^{3}$ in these groups.

However, an apparent reliance on the use of intravenous therapy is still reported, and this seems to be associated with lack of familiarity with oral rehydration techniques. ${ }^{4}$

Currently in emergency departments throughout Australasia, proscriptive guidelines are only available for intravenous fluid resuscitation in the severely dehydrated child. ${ }^{5}$ As yet, there is no consensus as to the best method of starting rehydration during a child's attendance at the emergency department with mild to moderate dehydration.

The objectives of this pilot study are to ascertain if the adoption of an oral rehydration protocol changes the admission rate, the total time spent in the emergency department, total time spent in hospital, or the number of unscheduled returns for children presenting to the emergency department with gastroenteritis.

\section{METHODS}

The study took place in an urban emergency department with an annual census of 43000 patients of which 12000 per annum are children aged less than 16 years. Anonymised data were extracted from the emergency department computer information system (HAS Edis, Hospital Administrative Software Solutions Pty, Sydney, Aus). All notes for patients aged less than 15 years of age with a discharge diagnosis of vomiting, diarrhoea, or gastroenteritis were prospectively analysed over a two month period before the adoption of an oral rehydration protocol. Patients that did not wait for review were excluded from analysis.

Only children triaged as being in Australasian National Triage categories three and four were included in the study. Patients in triage categories three and four are recommended to undergo physician supervised intervention inside 30 and 90 minutes respectively and thus represent comparatively stable patients who do not require immediate treatments. Patients were excluded from this study if their triage category was initially or subsequently upgraded to triage category two or above thus mandating physician intervention inside 10 minutes. ${ }^{6}$

During the initial "baseline" data collection period there was no structured departmental response for children presenting with gastroenteritis to our department. In addition there were no standing orders concerning the use of rehydration therapy at triage by the triage nursing staff. A subsequent two week lead in period was designated and during this time extensive teaching and information packages concerning oral rehydration were made available to all members of both medical and nursing staff. No data were analysed from this lead in period. A further two month prospective sampling period then occurred during which the rehydration protocol was adopted. The triage nurse, before physician assessment started the oral rehydration protocol. All therapies were undertaken within the emergency department, as no short stay facility was available

The two groups identified were matched for age and triage category on presentation.

Data were collected for admission rates, total time spent in hospital, the total time spent in the emergency department, and the number of unscheduled returns to the emergency department within 48 hours of initial attendance. Total time in the emergency department and total time in hospital were 
calculated for patients who underwent medical review therefore excluding those patients who did not wait for review. The oral rehydration protocol used is available on line (http://www.emjonline.com/supplemental).

A fridge stocked daily with commercially available flavoured electrolyte ice blocks and flavoured electrolyte drinks was provided at triage in the emergency department during the protocol trial period. Patients were also allowed to use fluids provided by themselves.

Results were analysed using Fisher's exact test for categorised data and Wilcoxon's test for continuous nonparametric data.

A power study was not undertaken in this, the pilot study phase.

\section{RESULTS}

A total of 40 children aged less than 15 years were identified in the pre-protocol period and 39 children in the postprotocol period. There was complete data capture from both groups. The mean age in the pre protocol group was 3.7 years ( $95 \% \mathrm{CI}=2.3$ to 4.31 , range $0-12$ years). The post-protocol group had a mean age of 3.6 years $(95 \% \mathrm{CI}=2.0$ to 4.0 , range $0-11$ years). In the pre-protocol group 32\% (13 of 40 ) were in triage category three and 68\% (27 of 40 ) in category four. In the post-protocol group $31 \%$ (12 of 39) were in triage category three and 69\% (27 of 39) in category four. There was no significant difference between the groups for age or triage category on presentation (both $\mathrm{p}>0.1$ )

Admission rates to the paediatric inpatient unit fell from $22.5 \%$ (9 of 40 ) during the pre-protocol period to $5.1 \%$ ( 2 of $39)$ for the post-protocol period. This change was significant $(\mathrm{p}=0.048)$.

In terms of total time spent in the hospital, inclusive of time in the emergency department, there was a decrease from a mean of 7 hours 54 minutes (95\% CI 3 hours 27 minutes to 21 hours 12 minutes) to a mean of 2 hours 17 minutes (95\% CI 1 hour 11 minutes to 2 hours 42 minutes) after the adoption of the protocol. This again was significant on testing $(p=0.017)$. The median total hospital time was 1 hour 45 minutes pre protocol and 1 hour 27 minutes after inception of the protocol.

The total time spent in the emergency department rose from a mean of 1 hour 25 minutes in the pre-protocol period (95\% CI 1 hour 1 minute to 1 hour 29 minutes) to a mean of 1 hour 35 minutes in the post-protocol period (95\% CI 1 hour 10 minutes to 1 hour 59 minutes). This rise was not significant $(p=0.3)$. The median time spent in the emergency department during the pre-protocol period was 1 hour 10 minutes and in the post-protocol period was 1 hour 22 minutes.

In respect to unscheduled returns within 48 hours of initial presentation, these fell from $7.5 \%$ ( 3 of 40 ) in the preprotocol period to $2.6 \%$ ( 1 of 39 ) in the post-protocol period. There was no significant difference between the groups $(\mathrm{p}=0.3)$.

\section{DISCUSSION}

This pilot study show that use of an oral fluid rehydration protocol for children presenting with uncomplicated gastroenteritis to the paediatric emergency department significantly reduces the need for admission and total time spent by children in hospital. As a result hospital costs can be decreased and pressure on inpatient beds reduced. These results were achieved within a comparatively short time period and required minimal financial input. Considerable teaching resources were however used to promote the use of oral rehydration therapy within the emergency department and this occurred during routine "in service" training periods.

Actual patient satisfaction was not directly measured during this study. However, the rate of unscheduled return did not significantly change after the adoption of the rehydration protocol. Although not directly measuring satisfaction, these results suggest acceptance of the oral rehydration protocol by parents and carers or indeed may represent an element of parental education.

This study has several weaknesses. The pilot study by its very nature contained small numbers leading to an increased risk of potential type II errors. Furthermore, only limited patient details were available with which to compare the preprotocol and post-protocol groups.

A variety of fluid administration methods were used, namely oral and frozen fluids. Frozen fluid therapy has previously been suggested to be superior to oral fluid therapy. ${ }^{7}$ The comparatively small numbers analysed in our study precluded sub-group analysis of the different fluid therapy types. Instead, all fluid therapies used were analysed as one.

Identification of cases was performed using a defined search strategy for the HAS Edis computer system (Hospital Administrative Software Solutions Pty, Sydney, Aus). The success of such a strategy depends on the accuracy of the discharge diagnosis coding. All patients with a diagnosis of gastroenteritis, vomiting, or diarrhoea were identified but other potentially relevant presentations, which were not correctly coded, may not have been identified.

The results presented have been achieved over a comparatively short time frame. A repeat study with substantially larger numbers and improved collection of clinical parameters is needed to prove if the presented results prove to be sustainable.

In conclusion, adoption of an oral rehydration protocol in the emergency department leads to a significant reduction in admission rates and total time in hospital without increasing the unscheduled return rate or time the patient spends in the emergency department.

\section{ACKNOWLEDGEMENTS}

The authors would like to acknowledge the Emergency Department of the Children's Hospital at Westmead, NSW for their assistance in the development of the rehydration protocol. The authors would also like to acknowledge the contribution of the National Institute for Clinical Studies Emergency Department collaborative group in the development of this study's methodology.

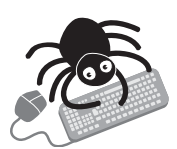

The oral rehydration protocol is available on line (http://www.emionline.com/supplemental).

\section{Authors' affiliations}

R Boyd, M Busuttil, P Stuart, Department of Emergency Medicine, Lyell McEwin Health Services, South Australia

Funding: none.

Conflicts of interest: none declared.

\section{REFERENCES}

1 Armon K, Stephenson T, MacFaul R, et al. Evidence and consensus based guideline for acute diarrhoea management. Arch Dis Child 2001;85:132-41.

2 Victoria CG, Bryce J, Fontaine O, et al. Reducing deaths from diarrhoea through oral rehydration therapy. Bull World Health Organ 2000;78:1246-55.

3 Gavin N, Merrick N, Davidson B. Efficacy of glucose-based oral rehydration therapy. Pediatrics 1996;98:45-51.

4 Ozuah PO, Avner JR, Stein RE. Oral rehydration, emergency physicians and practice parameters. Pediatrics 2002;109:259-61

5 Advanced Life Support. Advanced paediatric life support-the practical approach. London: BMJ Books, 2000.

6 Australasian College of Emergency Medicine. The Australian triage scale. Emerg Med 2002; 14:335-6.

7 Santucci K, Anderson C, Lewander W, et al. Frozen oral hydration as an alternative to conventional enteral fluids. Arch Pediatr Adolesc Med $1998 ; 152: 142-6$. 